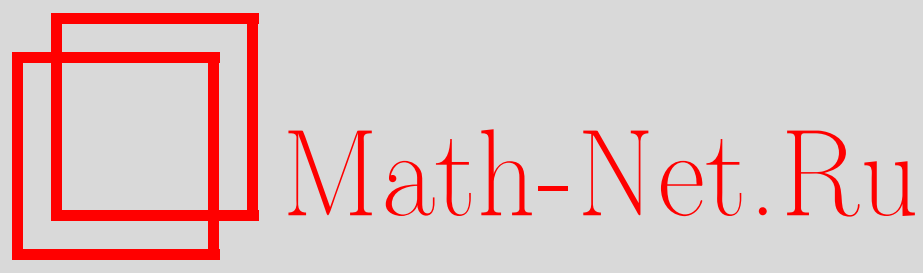

С. В. Сазонов, Н. В. Устинов, Векторные акустические солитоны при взаимодействии длинных и коротких волн в парамагнитном кристалле, ТМФ, 2014, том 178, номер 2, 230-254

DOI: https://doi.org/10.4213/tmf8560

Использование Общероссийского математического портала Math-Net.Ru подразумевает, что вы прочитали и согласны с пользовательским соглашением http: //www . mathnet.ru/rus/agreement

Параметры загрузки:

IP : 3.93 .64 .190

26 апреля 2023 г., 10:00:45

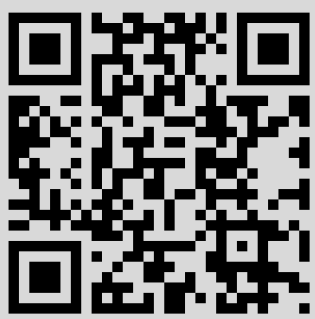




\title{
ВЕКТОРНЫЕ АКУСТИЧЕСКИЕ СОЛИТОНЫ ПРИ ВЗАИМОДЕЙСТВИИ ДЛИННЫХ И КОРОТКИХ ВОЛН В ПАРАМАГНИТНОМ КРИСТАЛЛЕ
}

\begin{abstract}
Исследовано распространение продольно-поперечного упругого импульса в статически деформированном кристалле, содержащем парамагнитные примеси и помещенном во внешнее магнитное поле. Выведена система трех нелинейных волновых уравнений, описывающая в рамках квазирезонансного приближения взаимодействие импульса с парамагнитными примесями в условиях геометрии Фарадея. При этом считается, что поперечные компоненты импульса, вызывающие квантовые переходы, обладают несущими частотами и являются коротковолновыми (акустическими), в то время как продольная компонента не имеет несущей частоты и является длинноволновой. Показано, что в случае равновесного начального распределения населенностей квантовых уровней парамагнитных примесей взаимодействие между продольной и поперечными компонентами будет слабым, из-за чего импульс оказывается сугубо поперечным, а его динамику описывает система Манакова. При неравновесном начальном распределении населенностей возможно достижение условий эффективного взаимодействия между всеми компонентами упругого импульса, а их нелинейная динамика описывается векторным обобщением уравнений Захарова. В случае однонаправленного распространения импульсов эти уравнения сведены к векторной системе Ядзимы-Ойкавы. Показано, что полученную систему уравнений и ее вариант с произвольным числом коротковолновых компонент можно проинтегрировать методом обратной задачи рассеяния. Построены бесконечные иерархии решений (в том числе решений на нетривиальном фоне) векторной системы Ядзимы-Ойкавы. Рассмотрены стационарная (комплекснозначная система Гарнье) и автомодельная редукции этой системы, также допускающие представление в виде условия совместности.
\end{abstract}

Ключевые слова: солитон, нелинейные интегрируемые уравнения, резонанс длинных и коротких волн.

DOI: $10.4231 / \operatorname{tmf} 8560$

*Национальный исследовательский центр "Курчатовский институт", Москва, Россия. E-mail: sazonov.sergey@gmail.com

${ }^{\dagger}$ Калининградский филиал Московского государственного университета путей сообщения, Калининград, Россия. E-mail: n_ustinov@mail.ru 


\section{1. ВВЕДЕНИЕ}

Вопросы интегрируемости нелинейных уравнений и систем занимают одно из центральных мест в теоретической и математической физике [1]. Наличие у таких уравнений физических приложений увеличивает интерес к подобным исследованиям многократно. На сегодняшний день известно богатое множество нелинейных интегрируемых уравнений, описывающих различные явления в разных областях физики.

С интегрируемостью нелинейных волновых уравнений тесно связано такое понятие, как солитон. Пожалуй, наиболее привлекательным с физической точки зрения свойством этого типа локализованных решений нелинейных уравнений является устойчивость, проявляющаяся в восстановлении формы солитона после взаимодействия с себе подобными, а также с любыми другими локализованными (и даже нелокализованными [2]) структурами. Строгое определение солитонного решения, объясняющее такое его уникальное свойство, дается в терминах метода обратной задачи рассеяния (МО3Р) [3], [4].

Наиболее часто системы, интегрируемые в рамках МОЗР, возникают в гидродинамике, физике плазмы, оптике, акустике, физике твердого тела. Одним из обстоятельств, привлекающих многих исследователей к солитонам, является то, что они, будучи различными по физической природе, описываются порой одними и теми же или похожими друг на друга уравнениями. Это позволяет считать солитоны универсальными физическими объектами. Что касается богатого математического содержания теории солитонов, то к настоящему времени оно нашло отражение в едва ли обозримом числе монографий.

Часто возникают физические ситуации, когда нелинейность и дисперсия среды, приводящие к образованию солитонов, оказываются относительно слабыми. В таких случаях обычно используют разложения по степеням рассматриваемого возмущения, называемым степенями нелинейности, и ограничиваются его низшими членами. В изотропных средах низшей нелинейностью является кубическая в силу симметрии задачи. Если исследуемое возмущение в такой среде представляет собой квазимонохроматический волновой пакет, то динамика его огибающей при учете нелинейности и групповой дисперсии в минимальных порядках описывается нелинейным уравнением Шредингера (НУШ) [5], которое принадлежит к классу уравнений, интегрируемых в рамках МОЗР [6]. Если же в этой задаче учесть нелинейное взаимодействие между волновыми пакетами с разными несущими частотами, то при определенных условиях можно прийти к системе Манакова - интегрируемому векторному (двухкомпонентному) обобщению НУШ [7]. Что касается анизотропных сред, то в них низшей нелинейностью является уже квадратичная. По этой причине в анизотропных средах возможна, в числе прочего, генерация низкочастотного сигнала на частоте, равной разности частот двух монохроматических волн. Кроме того, такая генерация может происходить при распространении квазимонохроматического волнового пакета. Для такого пакета в спектре шириной $\delta \omega \sim 1 / \tau_{\mathrm{p}}$, где $\tau_{\mathrm{p}}-$ его длительность, изначально содержатся частоты, на разности которых способны порождаться длинноволновые возмущения. При этом исходный сигнал, подаваемый 
на вход в анизотропную среду и имеющий несущую частоту $\omega$, является коротковолновым, т. е. $\omega \tau_{\mathrm{p}} \gg 1$. В результате приходим к задаче о связанном нелинейном распространении длинно-коротковолновых возмущений.

Так как спектр коротковолнового пакета сплошной, имеется непрерывное множество разностных частот. Поэтому ширина спектра генерируемого длинноволнового сигнала и его центральная частота являются величинами одного порядка и составляют примерно $1 / \tau_{\mathrm{p}}$. При этом длительность сигнала, порождаемого в среде, не может превышать длительность $\tau_{\mathrm{p}}$ входного импульса. Таким образом, генерируемый длинноволновый сигнал является широкополосным в спектральном смысле и содержит внутри себя всего лишь около одного периода колебаний. Иногда говорят, что спектр длинноволнового сигнала обладает свойствами суперконтинуума [8].

Помимо квадратичной нелинейности, для генерации длинноволнового сигнала с помощью коротковолнового необходимо выполнение условий синхронизма, которые можно вывести на основе законов сохранения энергии и импульса для квазичастиц при элементарных актах рассеяния, не конкретизируя природу квазичастиц. Пусть $\omega(\mathbf{k})$ и $\widetilde{\omega}(\mathbf{q})$ - законы дисперсии среды в коротко- и длинноволновой областях соответственно, т.е. зависимости частот $\omega$ и $\widetilde{\omega}$ от волновых векторов $\mathbf{k}$ и $\mathbf{q}$. Закон сохранения энергии-импульса при генерации длинноволновой квазичастицы в результате распада коротковолновой запишется в виде $\omega(\mathbf{k})=\omega(\mathbf{k}-\mathbf{q})+\widetilde{\omega}(\mathbf{q})$. Учитывая малость вектора $\mathbf{q}$ в сравнении с $\mathbf{k}$, запишем приближенное равенство

$$
\omega(\mathbf{k}-\mathbf{q}) \approx \omega(\mathbf{k})-\frac{\partial \omega(\mathbf{k})}{\partial \mathbf{k}} \cdot \mathbf{q}=\omega-\mathbf{v}_{\mathrm{g}} \cdot \mathbf{q},
$$

где $\mathbf{v}_{\mathrm{g}}=\partial \omega(\mathbf{k}) / \partial \mathbf{k}=\mathbf{v}_{\mathrm{g}}(\omega)$ - групповая скорость на несущей частоте $\omega$. В результате приходим к соотношению $\widetilde{\omega}=\mathbf{v}_{\mathrm{g}} \cdot \mathbf{q}$, которое можно записать в виде черенковского условия $\cos \theta=v_{\mathrm{ph}} / v_{\mathrm{g}}$, где $v_{\mathrm{ph}}=\widetilde{\omega} / q-$ значение фазовой скорости в длинноволновой области спектра, $\theta$ - угол между направлениями распространения входного коротковолнового и генерируемого длинноволнового сигналов. Очевидно, что наиболее эффективно процесс генерации длинноволнового сигнала и его взаимодействия с коротковолновым импульсом происходит в коллинеарном режиме (при $\theta=0$ ). Тогда условие синхронизма примет вид

$$
v_{\mathrm{g}}(\omega)=v_{\mathrm{ph}}(\widetilde{\omega}) .
$$

Это равенство называется в теории нелинейных волн условием резонанса Захарова-Бенни (РЗБ) [4].

В длинноволновой области спектра часто можно пренебречь дисперсией, т. е. счи-

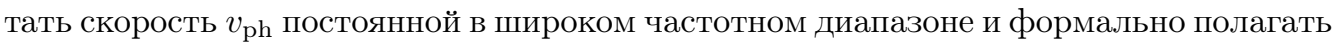
в условии (1) $\widetilde{\omega}=0$.

Впервые возможность образования связанных состояний длинно-коротковолнового типа была рассмотрена в задаче физики плазмы [9]. Соответствующая система двух нелинейных волновых уравнений получила название уравнений Захарова. Ее однонаправленный вариант, известный как система Ядзимы-Ойкавы (ЯО), оказался интегрируемым с помощью МОЗР [10]. Это обстоятельство позволило существенно продвинуться в понимании особенностей совместной нелинейной динамики длинных и коротких волн. В дальнейшем системы уравнений, описывающие длинно- 
коротковолновое взаимодействие, появлялись в гидродинамике [11], теории ферромагнетизма [12], оптике [13], акустике [14], опто-акустике [15].

Что касается прикладного аспекта использования взаимодействующих нелинейных волн разных частотных диапазонов, то в последнее время он наиболее четко просматривается в оптических методах генерации терагерцового излучения [16], которые находят свое применение в системах безопасности, биомедицине, формировании изображений и т.д. [8], [17]. В этом случае оптический импульс является коротковолновым, а генерируемый терагерцовый - длинноволновым.

Весьма часто нелинейные оптические явления находят с течением времени соответствия в задачах нелинейной акустики [16], [18]-[20]. Иногда, однако, сначала обнаруживается акустический эффект, а затем - его оптический аналог. Так, в работе [21] была показана возможность генерации ультразвукового (длинноволнового) суперконтинуума с помощью двухчастотного гиперзвукового (коротковолнового) воздействия. Были найдены условия, при которых равенство (1) выполняется сразу для обеих несущих частот. Соответствующая нелинейная динамика компонент поля упругости описывается системой нелинейных уравнений, обобщающей систему ЯО на случай двух коротковолновых компонент. Интегрируемость этой векторной системы ЯО в рамках МОЗР установить не удалось. Чуть позже в оптике, в задаче о квазирезонансном взаимодействии оптических импульсов с несимметричными квантовыми объектами, было выведено еще одно двухкомпонентное обобщение системы ЯО, которое оказалось интегрируемым в рамках МОЗР [22]. Следуя, в числе прочего, логике поиска оптико-акустических соответствий, можно поставить вопрос о нахождении физической реализации и математическом исследовании векторного интегрируемого обобщения системы ЯО в акустике. Этому посвящена настоящая работа.

Статья построена следующим образом. В разделе 2 выводится система волновых и материальных уравнений, которая описывает распространение продольно-поперечных упругих импульсов в парамагнитном кристалле параллельно статической деформации и магнитному полю. Раздел 3 посвящен исключению материальных переменных в квазирезонансном приближении и выводу таким образом системы нелинейных уравнений только для полевых компонент. Здесь же определяются условия, при которых взаимодействие между продольной (длинноволновой) и поперечными (коротковолновыми) импульсными составляющими является наиболее эффективным. В разделе 4 показано, что векторное обобщение системы ЯО, описывающее такое взаимодействие и имеющее, вообще говоря, произвольное число коротковолновых компонент, интегрируемо в рамках МОЗР. Построена бесконечная иерархия решений такого обобщения, которая включает в себя многосолитонные решения и решения на фоне конечнозонных потенциалов. Раздел 5 посвящен стационарной и автомодельной редукциям векторной системы ЯО. Возникающие при этом системы обыкновенных дифференциальных уравнений также представимы в виде условия совместности переопределенных систем линейных уравнений. В разделе 6 подведены основные итоги работы и намечены некоторые перспективные исследования. 


\section{2. СИСТЕМА ВОЛНОВЫХ И МАТЕРИАЛЬНЫХ УРАВНЕНИЙ}

Рассмотрим кубический кристалл, содержащий в качестве примесей парамагнитные ионы, обладающие эффективным спином $S=1$. Пусть вдоль оси $z$, совпадающей с одной из осей симметрии четвертого порядка, к кристаллу приложены статическая деформация величиной $\varepsilon_{0}$ и внешнее магнитное поле В. Параллельно этой же оси распространяется продольно-поперечный упругий импульс, взаимодействующий за счет спин-фононной связи с парамагнитными примесями. Часто такую конфигурацию называют геометрией Фарадея.

Обычно спин-фононное взаимодействие осуществляется посредством механизма Ван Флека [23]. Акустическая волна, проходящая через кристалл, вызывает в нем локальные деформации, которые модулируют градиенты внутрикристаллического электрического поля. Последние как вызывают электрические квадрупольные переходы между квантовыми зеемановскими подуровнями парамагнитных примесей, так и динамически, за счет квадрупольного эффекта Штарка, смещают частоты переходов. При этом наиболее сильное взаимодействие с колебаниями кристаллической решетки могут испытывать парамагнитные ионы с эффективным спином $S=1$. Такими ионами являются, например, ионы $\mathrm{Fe}^{2+}$ или $\mathrm{Ni}^{2+}$, внедренные в кубический кристалл $\mathrm{MgO}$ [23], [24].

Для возникновения эффективного спин-фононного взаимодействия должна быть достаточно большой разность населенностей подуровней, образовавшихся в результате зеемановского и штарковского расщеплений квантовых уровней эффективного спина. Пусть $\omega_{0}$ - характерная частота расщепления. Тогда соответствующая энергия $\hbar \omega_{0}$ должна превышать тепловую энергию $k_{\mathrm{B}} T$ (здесь $k_{\mathrm{B}}-$ постоянная Больцмана, $T$ - абсолютная температура). Взяв $\omega_{0} \sim 10^{11} \mathrm{c}^{-1}$ [18], [23], [24], найдем, что $T \sim 1 \mathrm{~K}$. По этой причине в экспериментальных условиях парамагнитные кристаллы помещают в жидкий гелий. Кроме того, при таких температурах можно пренебречь затуханием гиперзвука, вызванным фонон-фононным взаимодействием, рассеянием на дефектах и т. д. [23].

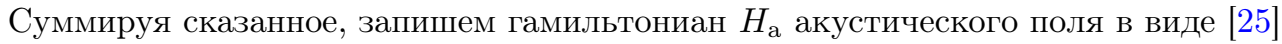

$$
H_{\mathrm{a}}=\frac{1}{2} \int\left\{\frac{p_{x}^{2}+p_{y}^{2}+p_{z}^{2}}{\rho}+\rho a_{\|}^{2}\left(\frac{\partial u_{z}}{\partial z}\right)^{2}+\rho a_{\perp}^{2}\left[\left(\frac{\partial u_{x}}{\partial z}\right)^{2}+\left(\frac{\partial u_{y}}{\partial z}\right)^{2}\right]\right\} d \mathbf{r}
$$

где $u_{j}$ и $p_{j}, j=x, y, z,-$ декартовы компоненты векторов $\mathbf{u}$ смещений узлов кристаллической решетки и соответствующих им плотностей импульсов $\mathbf{p}, \rho-$ средняя плотность среды, $a_{\|}$и $a_{\perp}-$ скорости продольного и поперечного звуков соответственно в отсутствие парамагнитных примесей; интегрирование в (2) ведется по всему объему кристалла.

Дополним (2) оператором Гамильтона $\widehat{H}_{\mathrm{s}}$, который связывает эффективный спин с магнитным полем, а также с упомянутой выше статической деформацией и компонентами тензора динамической деформации [26]:

$$
\begin{aligned}
\widehat{H}_{\mathrm{s}}= & \hbar \omega_{z} \widehat{S}_{z}+G_{\|} \widehat{S}_{z}^{2} \varepsilon_{0}+G_{\|} \widehat{S}_{z}^{2} \frac{\partial u_{z}}{\partial z}+ \\
& +\frac{G_{\perp}}{2}\left[\left(\widehat{S}_{x} \widehat{S}_{z}+\widehat{S}_{z} \widehat{S}_{x}\right) \frac{\partial u_{x}}{\partial z}+\left(\widehat{S}_{y} \widehat{S}_{z}+\widehat{S}_{z} \widehat{S}_{y}\right) \frac{\partial u_{y}}{\partial z}\right]
\end{aligned}
$$




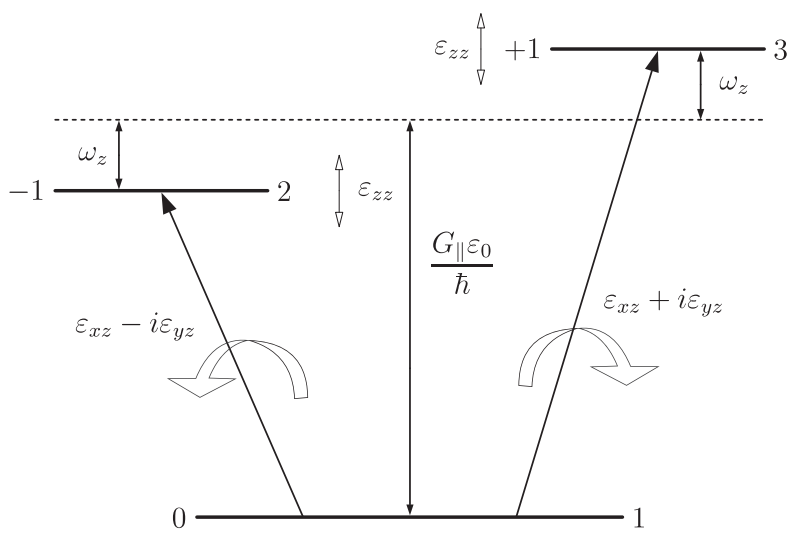

Рис. 1. Квадрупольное $G_{\|} \varepsilon_{0} / \hbar$ и зеемановское $\omega_{z}$ расщепления квантового состояния эффективного спина $S=1$ на три подуровня. Номера образовавшихся подуровней обозначены справа от них, а проекции $S_{z}$ - слева. Циркулярно-поляризованные в противоположных направлениях поперечные компоненты поля деформации $\varepsilon_{x z}+i \varepsilon_{y z}$ и $\varepsilon_{x z}-i \varepsilon_{y z}$ вызывают соответственно переходы $1 \leftrightarrow 3$ и $1 \leftrightarrow 2$. Продольная компонента $\varepsilon_{z z}$ динамически сдвигает частоты обоих разрешенных переходов.

Здесь $\omega_{z}$ - частота зеемановского расщепления спиновых подуровней, снимающего вырождение по проекции $S_{z}$ эффективного спина на ось $z, G_{\|}$и $G_{\perp}$ - постоянные спин-фононного взаимодействия, связывающие эффективный спин соответственно с компонентами тензора продольной и поперечной деформации, а $\widehat{S}_{x}, \widehat{S}_{y}$ и $\widehat{S}_{z}-$ спиновые матрицы, имеющие в представлении собственных функций оператора $\widehat{S}_{z}$ вид

$$
\widehat{S}_{x}=\frac{1}{\sqrt{2}}\left(\begin{array}{ccc}
0 & 0 & 1 \\
0 & 0 & 1 \\
1 & 1 & 0
\end{array}\right), \quad \widehat{S}_{y}=\frac{i}{\sqrt{2}}\left(\begin{array}{ccc}
0 & 0 & -1 \\
0 & 0 & 1 \\
1 & -1 & 0
\end{array}\right), \quad \widehat{S}_{z}=\left(\begin{array}{ccc}
1 & 0 & 0 \\
0 & -1 & 0 \\
0 & 0 & 0
\end{array}\right) .
$$

Будем считать, что основной вклад в расщепление спиновых подуровней вносит квадрупольный эффект Штарка, снимающий вырождение по модулю проекции $S_{z}$ (второе слагаемое в правой части равенства (3)). При этом зеемановское расщепление (первое слагаемое там же), за счет которого снимается вырождение по величине $S_{z}$, вносит небольшое возмущение. Тогда вид спиновых матриц (4) соответствует нумерации квантовых уровней снизу вверх по энергии, т. е. проекциям $S_{z}=0,-1,+1$ отвечают номера 1, 2, 3 квантовых уровней (см. рис. 1).

Исследование самосогласованной динамики эффективных спинов и поля упругости будем проводить, применяя полуклассический подход. Согласно этому подходу поведение эффективных спинов описывается квантовомеханическим уравнением для соответствующей матрицы плотности $\hat{\rho}$ :

$$
i \hbar \frac{\partial \hat{\rho}}{\partial t}=\left[\widehat{H}_{\mathrm{s}}, \hat{\rho}\right]
$$


а эволюция поля упругости - классическими уравнениями Гамильтона для сплошной среды [27]:

$$
\frac{\partial \mathbf{u}}{\partial t}=\frac{\delta}{\delta \mathbf{p}}\left(H_{\mathrm{a}}+\int n\left\langle\widehat{H}_{\mathrm{s}}\right\rangle d \mathbf{r}\right), \quad \frac{\partial \mathbf{p}}{\partial t}=-\frac{\delta}{\delta \mathbf{u}}\left(H_{\mathrm{a}}+\int n\left\langle\widehat{H}_{\mathrm{s}}\right\rangle d \mathbf{r}\right),
$$

где $\left\langle\widehat{H}_{\mathrm{s}}\right\rangle=\operatorname{Tr}\left(\hat{\rho} \widehat{H}_{\mathrm{s}}\right)$ - квантовое среднее гамильтониана $(3), n$ - плотность эффективных спинов. Используя соотношения (2)-(6), придем к следующей системе материальных и волновых уравнений:

$$
\begin{aligned}
& \frac{\partial \rho_{13}}{\partial t}=i\left(\omega_{31}+U\right) \rho_{13}-i \Omega\left(\rho_{33}-\rho_{11}\right)+i \Omega^{*} \rho_{23}, \\
& \frac{\partial \rho_{12}}{\partial t}=i\left(\omega_{21}+U\right) \rho_{12}+i \Omega^{*}\left(\rho_{22}-\rho_{11}\right)-i \Omega \rho_{23}^{*}, \\
& \frac{\partial \rho_{23}}{\partial t}=i \omega_{23} \rho_{23}+i \Omega \rho_{12}^{*}+i \Omega \rho_{13}, \\
& \frac{\partial \rho_{33}}{\partial t}=i\left(\Omega \rho_{13}^{*}-\Omega^{*} \rho_{13}\right), \\
& \frac{\partial \rho_{22}}{\partial t}=i\left(\Omega \rho_{12}-\Omega^{*} \rho_{12}^{*}\right)
\end{aligned}
$$

и

$$
\begin{aligned}
& \frac{\partial^{2} \Omega}{\partial t^{2}}-a_{\perp}^{2} \frac{\partial^{2} \Omega}{\partial z^{2}}=\frac{n G_{\perp}^{2}}{4 \hbar \rho} \frac{\partial^{2}}{\partial z^{2}}\left(\rho_{13}-\rho_{12}^{*}\right), \\
& \frac{\partial^{2} U}{\partial t^{2}}-a_{\|}^{2} \frac{\partial^{2} U}{\partial z^{2}}=-\frac{n G_{\|}^{2}}{\hbar \rho} \frac{\partial^{2} \rho_{11}}{\partial z^{2}} .
\end{aligned}
$$

Здесь $\omega_{31}=G_{\|} \varepsilon_{0} / \hbar+\omega_{z}$ и $\omega_{21}=G_{\|} \varepsilon_{0} / \hbar-\omega_{z}$ - частоты разрешенных квантовых переходов $1 \leftrightarrow 3$ и $1 \leftrightarrow 2$ соответственно (см. рис. 1 ), $\omega_{32}=2 \omega_{z}$ - частота запрещенного перехода $2 \leftrightarrow 3$,

$$
U=\frac{G_{\|} \varepsilon_{z z}}{\hbar}, \quad \Omega=\frac{G_{\perp}}{\hbar} \frac{\varepsilon_{x z}+i \varepsilon_{y z}}{\sqrt{2}},
$$

а продольная $\varepsilon_{z z}$ и поперечные $\varepsilon_{x z}, \varepsilon_{y z}$ компоненты тензора деформации поля упругости выражаются через компоненты вектора смещений u следующим образом:

$$
\varepsilon_{z z}=\frac{\partial u_{z}}{\partial z}, \quad \varepsilon_{x z}=\frac{1}{2} \frac{\partial u_{x}}{\partial z}, \quad \varepsilon_{y z}=\frac{1}{2} \frac{\partial u_{y}}{\partial z} .
$$

Отметим, что населенности задействованных в динамике квантовых уровней подчиняются условию $\rho_{11}+\rho_{22}+\rho_{33}=1$.

Из уравнений (7) следует, что в геометрии Фарадея поперечные компоненты поля деформации вызывают квантовые переходы $1 \leftrightarrow 3$ и $1 \leftrightarrow 2$, в то время как продольная составляющая динамически, за счет квадрупольного эффекта Штарка, смещает частоты этих переходов.

Для дальнейшего упрощения самосогласованной системы уравнений (7)-(9) представим комплексную поперечную компоненту поля акустического импульса и недиагональные элементы матрицы плотности в виде

$$
\begin{gathered}
\Omega=\Omega_{+} e^{i \omega_{+}\left(t-z / a_{\perp}\right)}-\Omega_{-}^{*} e^{-i \omega_{-}\left(t-z / a_{\perp}\right)} \\
\rho_{13}=R_{13} e^{i \omega_{+}\left(t-z / a_{\perp}\right)}, \quad \rho_{12}=R_{12} e^{i \omega_{-}\left(t-z / a_{\perp}\right)}, \quad \rho_{23}=R_{23} e^{i\left(\omega_{+}-\omega_{-}\right)\left(t-z / a_{\perp}\right)},
\end{gathered}
$$


где $\Omega_{+}, \Omega_{-}, R_{13}, R_{12}, R_{23}$ - огибающие, медленно меняющиеся на протяжении периодов, которые соответствуют несущим частотам $\omega_{+}, \omega_{-}$и $\omega_{+}-\omega_{-}$.

Подставив соотношения (10), (11) в уравнения (7) и отбросив слагаемые, осциллирующие на суммарной частоте $\omega_{+}+\omega_{-}$, придем к материальным уравнениям для огибающих

$$
\begin{aligned}
& \frac{\partial R_{13}}{\partial t}=i\left(\Delta_{31}+U\right) R_{13}-i \Omega_{+}\left(\rho_{33}-\rho_{11}\right)+i \Omega_{-} R_{23}, \\
& \frac{\partial R_{12}}{\partial t}=i\left(\Delta_{21}+U\right) R_{12}-i \Omega_{-}\left(\rho_{22}-\rho_{11}\right)+i \Omega_{+} R_{23}^{*}, \\
& \frac{\partial R_{23}}{\partial t}=i\left(\Delta_{31}-\Delta_{21}\right) R_{23}+i \Omega_{+} R_{12}^{*}-i \Omega_{-}^{*} R_{13},
\end{aligned}
$$

где $\Delta_{31}=\omega_{31}-\omega_{+}, \Delta_{21}=\omega_{21}-\omega_{-}$- отстройки несущих частот от резонансов с переходами $1 \leftrightarrow 3$ и $1 \leftrightarrow 2$. При этом уравнения для диагональных элементов матрицы плотности примут вид

$$
\frac{\partial \rho_{33}}{\partial t}=i\left(\Omega_{+} R_{13}^{*}-\Omega_{+}^{*} R_{13}\right), \quad \frac{\partial \rho_{22}}{\partial t}=i\left(\Omega_{-} R_{12}^{*}-\Omega_{-}^{*} R_{12}\right) .
$$

Из уравнения (8), используя приближение медленно меняющихся огибающих [28], получим

$$
\frac{\partial \Omega_{+}}{\partial z}+\frac{1}{a_{\perp}} \frac{\partial \Omega_{+}}{\partial t}=i \frac{n G_{\perp}^{2} \omega_{+}}{8 \hbar \rho a_{\perp}^{3}} R_{13}, \quad \frac{\partial \Omega_{-}}{\partial z}+\frac{1}{a_{\perp}} \frac{\partial \Omega_{-}}{\partial t}=i \frac{n G_{\perp}^{2} \omega_{-}}{8 \hbar \rho a_{\perp}^{3}} R_{12} .
$$

Видно, что циркулярно-поляризованные огибающие $\Omega_{+}$и $\Omega_{-}$возбуждают соответственно переходы $1 \leftrightarrow 3$ и $1 \leftrightarrow 2$. Однако эти переходы не являются независимыми и влияют друг на друга через населенность $\rho_{11}$ основного спинового подуровня и огибающую когерентности $R_{23}$.

Система уравнений (12)-(16), (9) описывает нелинейное взаимодействие продольно-поперечного акустического импульса с парамагнитными примесями, содержащимися в кубическом кристалле. В рассматриваемой здесь геометрии Фарадея две противоположно вращающиеся циркулярно-поляризованные поперечные компоненты акустического импульса являются коротковолновыми, тогда как продольная компонента - длинноволновой.

\section{3. ИСКЛЮЧЕНИЕ МАТЕРИАЛЬНЫХ ПЕРЕМЕННЫХ. ВЕКТОРНЫЕ ОБОБЩЕНИЯ СИСТЕМ ЗАХАРОВА И ЯО}

Поставим вопрос об исключении материальных переменных из системы уравнений (12)-(16), (9) и выводе таким образом системы нелинейных волновых уравнений, описывающих взаимодействие длинных и коротких волн. При решении этого вопроса будем считать, что выполняются квазирезонансные условия [29]

$$
\mu=\left(\Delta_{21} \tau_{\mathrm{p}}\right)^{-1} \sim\left(\Delta_{31} \tau_{\mathrm{p}}\right)^{-1} \ll 1,
$$

где $\tau_{\mathrm{p}}$ - длительность импульса.

Физическое содержание наложенных условий заключается в следующем. Так как спектральная ширина импульса $\delta \omega$ имеет порядок величины $1 / \tau_{\mathrm{p}}$, из условий $(17)$ 
следует, что она мала по сравнению с отстройками его несущих частот от резонансных частот. Поэтому в спектре импульса практически не содержатся резонансные фононы, и, следовательно, взаимодействие с парамагнитными ионами будет слабым. В соответствии с приведенными в разделе 1 рассуждениями в случае квазирезонанса следует ожидать, что в волновых уравнениях нелинейность будет иметь степенной характер.

При условиях (17) левые части уравнений (12) и (13) малы. Считая также, что $|U| \ll\left|\Delta_{21}\right|,\left|\Delta_{31}\right|$, из уравнения (12) имеем

$$
R_{13}=\frac{\rho_{33}-\rho_{11}}{\Delta_{31}} \Omega_{+}-\frac{w_{3}-w_{1}}{\Delta_{31}^{2}} U \Omega_{+}+\frac{\Omega_{-}}{\Delta_{31}} R_{23}-\frac{i}{\Delta_{31}} \frac{\partial R_{13}}{\partial t},
$$

где в последних двух слагаемых мы пренебрегли вкладом продольной компоненты импульса, а во втором положили населенности $\rho_{33}$ и $\rho_{11}$ равными их начальным значениям $w_{3}$ и $w_{1}$ соответственно. Такое допущение оправдано тем, что в условиях квазирезонанса изменение населенностей квантовых уровней незначительно.

Из соотношений (18) и (14) видно, что огибающая $R_{23}$ квадратична по степеням величин $\Omega_{+}$и $\Omega_{-}$. Следовательно, предпоследнее слагаемое в правой части равенства (18) формирует кубическую нелинейность, а главный, линейный, вклад вносит первое слагаемое. Тогда в нулевом приближении по $\mu$ из (18) следует, что

$$
R_{13}=\frac{w_{3}-w_{1}}{\Delta_{31}} \Omega_{+}
$$

Подставив это выражение в последнее слагаемое в правой части равенства (18), найдем выражение для $R_{13}$ в первом приближении по $\mu$ и т. д. Во втором приближении будем иметь

$$
R_{13}=\frac{\rho_{33}-\rho_{11}}{\Delta_{31}} \Omega_{+}-\frac{w_{3}-w_{1}}{\Delta_{31}^{2}} U \Omega_{+}+\frac{\Omega_{-}}{\Delta_{31}} R_{23}-i \frac{w_{3}-w_{1}}{\Delta_{31}^{2}} \frac{\partial \Omega_{+}}{\partial t}-\frac{w_{3}-w_{1}}{\Delta_{31}^{3}} \frac{\partial^{2} \Omega_{+}}{\partial t^{2}} .
$$

Выражение для $R_{12}$ во втором приближении по $\mu$ получается из (19) при помощи замен в индексах 3 на 2 и + на - . Учитывая только главные члены в этих разложениях, из уравнения (14) находим, что

$$
\begin{aligned}
\frac{\partial R_{23}}{\partial t}= & i\left(\Delta_{31}-\Delta_{21}\right) R_{23}+i\left(\frac{w_{2}-w_{1}}{\Delta_{21}}-\frac{w_{3}-w_{1}}{\Delta_{31}}\right) \Omega_{+} \Omega_{-}^{*}- \\
& -\frac{w_{2}-w_{1}}{\Delta_{21}^{2}} \Omega_{+} \frac{\partial \Omega_{-}^{*}}{\partial t}-\frac{w_{3}-w_{1}}{\Delta_{31}^{2}} \Omega_{-}^{*} \frac{\partial \Omega_{+}}{\partial t} .
\end{aligned}
$$

Собственные частоты $\omega_{31}$ и $\omega_{21}$ незначительно отличаются друг от друга,

$$
\omega_{31}-\omega_{21}=2 \omega_{z} \ll \omega_{31}, \omega_{21},
$$

поэтому ниже будем полагать начальные населенности спиновых подуровней 2 и 3 одинаковыми: $w_{3}=w_{2}$. Кроме того, отстройки обеих поперечных компонент импульса от резонанса будем считать равными, т. е. $\Delta_{31}=\Delta_{21}=\Delta$. Тогда интегрирование уравнения (20) с нулевыми начальными условиями при $t \rightarrow-\infty$ дает

$$
R_{23}=\frac{w_{1}-w_{2}}{\Delta^{2}} \Omega_{+} \Omega_{-}^{*}
$$


При этом соотношение (19) перепишется следующим образом:

$$
\begin{aligned}
R_{13}= & \frac{\rho_{33}-\rho_{11}}{\Delta} \Omega_{+}-\frac{w_{2}-w_{1}}{\Delta^{2}} U \Omega_{+}-\frac{w_{2}-w_{1}}{\Delta^{3}}\left|\Omega_{-}\right|^{2} \Omega_{+}- \\
& -i \frac{w_{2}-w_{1}}{\Delta^{2}} \frac{\partial \Omega_{+}}{\partial t}-\frac{w_{2}-w_{1}}{\Delta^{3}} \frac{\partial^{2} \Omega_{+}}{\partial t^{2}} .
\end{aligned}
$$

Соответствующее выражение для $R_{12}$ получается из представленного выше заменой $\rho_{33}$ на $\rho_{22}$ и взаимной заменой $\Omega_{+} \leftrightarrow \Omega_{-}$. Подставив эти выражения в (15) и проинтегрировав полученные равенства с учетом начальных условий $\rho_{33}, \rho_{22} \rightarrow w_{2}$ при $t \rightarrow-\infty$, получим

$$
\rho_{33}=w_{2}+\frac{w_{1}-w_{2}}{\Delta^{2}}\left|\Omega_{+}\right|^{2}, \quad \rho_{22}=w_{2}+\frac{w_{1}-w_{2}}{\Delta^{2}}\left|\Omega_{-}\right|^{2} .
$$

Приняв во внимание интеграл $\rho_{11}+\rho_{22}+\rho_{33}=w_{1}+2 w_{2}$, находим

$$
\rho_{11}=w_{1}-\frac{w_{1}-w_{2}}{\Delta^{2}}\left(\left|\Omega_{+}\right|^{2}+\left|\Omega_{-}\right|^{2}\right) .
$$

Тогда выражения для огибающих $R_{13}$ и $R_{12}$ примут вид

$$
\begin{aligned}
R_{13}= & -\frac{w_{1}-w_{2}}{\Delta} \Omega_{+}\left(1-\frac{U}{\Delta}\right)+2 \frac{w_{1}-w_{2}}{\Delta^{3}}\left(\left|\Omega_{+}\right|^{2}+\left|\Omega_{-}\right|^{2}\right) \Omega_{+}+ \\
& +i \frac{w_{1}-w_{2}}{\Delta^{2}} \frac{\partial \Omega_{+}}{\partial t}+\frac{w_{1}-w_{2}}{\Delta^{3}} \frac{\partial^{2} \Omega_{+}}{\partial t^{2}}, \\
R_{12}= & -\frac{w_{1}-w_{2}}{\Delta} \Omega_{-}\left(1-\frac{U}{\Delta}\right)+2 \frac{w_{1}-w_{2}}{\Delta^{3}}\left(\left|\Omega_{+}\right|^{2}+\left|\Omega_{-}\right|^{2}\right) \Omega_{-}+ \\
& +i \frac{w_{1}-w_{2}}{\Delta^{2}} \frac{\partial \Omega_{-}}{\partial t}+\frac{w_{1}-w_{2}}{\Delta^{3}} \frac{\partial^{2} \Omega_{-}}{\partial t^{2}} .
\end{aligned}
$$

Подстановка выражений (22) и (21) в уравнения (16) и (9) приводит к следующей нелинейной системе:

$$
\begin{aligned}
i\left(\frac{\partial \Phi_{ \pm}}{\partial z}+\frac{1}{v_{\mathrm{g}}} \frac{\partial \Phi_{ \pm}}{\partial t}\right) & =\beta \frac{\partial^{2} \Phi_{ \pm}}{\partial t^{2}}+\alpha U \Phi_{ \pm}+2 \beta\left(\left|\Phi_{+}\right|^{2}+\left|\Phi_{-}\right|^{2}\right) \Phi_{ \pm} \\
\frac{\partial^{2} U}{\partial z^{2}}-\frac{1}{a_{\|}^{2}} \frac{\partial^{2} U}{\partial t^{2}} & =2 a_{\|} b \frac{\partial^{2}}{\partial z^{2}}\left(\left|\Phi_{+}\right|^{2}+\left|\Phi_{-}\right|^{2}\right)
\end{aligned}
$$

где $\Phi_{ \pm}=\Omega_{ \pm} e^{-i \alpha_{ \pm} \Delta z}$, использованы обозначения

$$
\begin{gathered}
\alpha_{ \pm}=\frac{n G_{\perp}^{2} \omega_{ \pm}}{8 \hbar \rho a_{\perp}^{3}} \frac{w_{2}-w_{1}}{\Delta^{2}}, \quad \alpha=\frac{\alpha_{+}+\alpha_{-}}{2}=\frac{n G_{\perp}^{2} \omega}{8 \hbar \rho a_{\perp}^{3}} \frac{w_{2}-w_{1}}{\Delta^{2}}, \\
\omega=\frac{\omega_{+}+\omega_{-}}{2}, \quad \beta=\frac{\alpha}{\Delta}, \quad b=\frac{n G_{\|}^{2}}{2 \hbar \rho a_{\|}^{3}} \frac{w_{2}-w_{1}}{\Delta^{2}},
\end{gathered}
$$

а линейная групповая скорость $v_{\mathrm{g}}$ поперечных компонент импульса определяется выражением

$$
\frac{1}{v_{\mathrm{g}}}=\frac{1}{a_{\perp}}-\alpha=\frac{1}{a_{\perp}}\left(1-\frac{n G_{\perp}^{2} \omega}{8 \hbar \rho a_{\perp}^{2}} \frac{w_{2}-w_{1}}{\Delta^{2}}\right) .
$$


В уравнениях (23) мы пренебрегли различиями в коэффициентах нелинейности и дисперсии обеих поперечных компонент, положив в них $\omega_{+}=\omega_{-}=\omega$, в силу того что эти различия представляют собой разложения по малому параметру $\mu$.

Система уравнений (23), (24) описывает распространение продольно-поперечного акустического импульса через парамагнитный кристалл в режиме нелинейного взаимодействия между его поперечными (коротковолновыми) и продольной (длинноволновой) составляющими. Из этой системы видно, что продольная компонента $U$ может быть порождена поперечными составляющими $\Phi_{ \pm}$. В то же время продольная акустическая компонента не способна породить поперечную, если та отсутствует на входе в кристалл. Кроме того, из полученных уравнений следует, что $a_{\|}$имеет смысл фазовой скорости $v_{\mathrm{ph}}$ длинноволновой компоненты, фигурирующей в соотношении (1).

В кристаллах линейные скорости $a_{\|}$и $a_{\perp}$ продольного и поперечного звуков значительно отличаются друг от друга, причем всегда $a_{\|}>a_{\perp}[30]$. Как видно из формулы (25), в случае термодинамически равновесной начальной заселенности спиновых подуровней, когда $w_{1}>w_{2}$, групповая скорость поперечного звука становится меньше $a_{\perp}$. Поэтому условие (1) не может быть выполнено. В силу этого не может быть сколько-нибудь эффективной генерации длинноволновой компоненты посредством коротковолновых составляющих, и образование связанных состояний длиннокоротковолнового типа невозможно. Следовательно, в равновесном случае можно считать $U=0$. Тогда уравнения (23) переходят в хорошо известную интегрируемую систему Манакова [7].

Предположим теперь, что до воздействия акустическим импульсом внедренные в кристалл парамагнитные ионы находятся в термодинамически неравновесном состоянии: $w_{1}<w_{2}$. Тогда, как видно из формулы (25), мы имеем $v_{\mathrm{g}}>a_{\perp}$, и возникает принципиальная возможность удовлетворить условию (1), при котором поперечные компоненты могут эффективно генерировать продольный звук. Используя (25), перепишем равенство (1) для нашего случая в виде

$$
\frac{n G_{\perp}^{2} \omega}{8 \hbar \rho a_{\perp}^{2}} \frac{w_{2}-w_{1}}{\Delta^{2}}=\frac{a_{\|}-a_{\perp}}{a_{\|}} .
$$

Полагая $w_{2}=0.5, w_{1}=0,\left(a_{\|}-a_{\perp}\right) / a_{\|} \sim 0.1, \omega \sim 10^{11} \mathrm{c}^{-1}, \Delta \sim 10^{9} \mathrm{c}^{-1}, \rho \approx 1 \mathrm{r} / \mathrm{cm}^{3}$, $a_{\perp} \sim 3 \cdot 10^{5} \mathrm{~cm} / \mathrm{c}, G_{\perp} \sim 10^{-14}$ эрг [18], [23], [24], [30], получим для плотности парамагнитных примесей оценку $n \sim 10^{19} \mathrm{~cm}^{-3}$, составляющую примерно $0.01 \%$ от плотности атомов кристаллической решетки. При таких условиях $v_{\mathrm{g}}=a_{\|}$, и возможно образование связанных состояний длинно-коротковолнового типа. Уравнения (23), (24), описывающие этот процесс, можно назвать системой Манакова-Захарова.

Пусть теперь выполняется неравенство

$$
|U| \gg \frac{2}{|\Delta|}\left(\left|\Phi_{+}\right|^{2}+\left|\Phi_{-}\right|^{2}\right)
$$

В этом случае в уравнениях (23) можно пренебречь последними слагаемыми в правых частях. Тогда уравнения (23), (24) представляют собой векторное обобщение системы Захарова. 
В правой части волнового уравнения (24) содержатся нелинейные слагаемые, связанные с малым изменением населенности основного спинового подуровня (см. формулу (21)). Это позволяет ограничить рассмотрение акустическими импульсами, распространяющимися только в одном направлении. В соответствии с приближением однонаправленного распространения [4] введем локальное время $T=t-z / a_{\|}$ и "медленную" координату $Z=\eta z$, где $\eta$ - малый параметр, характеризующий величину правой части уравнения (24). Имеем

$$
\frac{\partial}{\partial t}=\frac{\partial}{\partial T}, \quad \frac{\partial}{\partial z}=-\frac{1}{a_{\|}} \frac{\partial}{\partial T}+\eta \frac{\partial}{\partial Z}, \quad \frac{\partial^{2}}{\partial z^{2}}=\frac{1}{a_{\|}^{2}} \frac{\partial^{2}}{\partial T}-\frac{2 \eta}{a_{\|}} \frac{\partial^{2}}{\partial T \partial Z} .
$$

В последнем соотношении мы пренебрегли слагаемым, квадратичным по $\eta$. Поступив также для правой части уравнения (24), положим

$$
\frac{\partial^{2}}{\partial z^{2}}=\frac{1}{a_{\|}^{2}} \frac{\partial^{2}}{\partial T^{2}}
$$

Тогда после интегрирования по $T$ и возвращения к исходной переменной $z$ получим для $U$ волновое уравнение первого порядка. Приняв также во внимание условие РЗБ $v_{\mathrm{g}}=a_{\|}$и оценку $(26)$, придем окончательно к следующей системе:

$$
\begin{aligned}
i \frac{\partial \Phi_{ \pm}}{\partial z} & =\beta \frac{\partial^{2} \Phi_{ \pm}}{\partial T^{2}}+\alpha U \Phi_{ \pm}, \\
\frac{\partial U}{\partial z} & =-b \frac{\partial}{\partial T}\left(\left|\Phi_{+}\right|^{2}+\left|\Phi_{-}\right|^{2}\right) .
\end{aligned}
$$

Полученная система уравнений представляет собой векторное (с двумя коротковолновыми компонентами) обобщение системы ЯО. Когда одна из коротковолновых компонент $\Phi_{+}$или $\Phi_{-}$тождественно равна нулю, эта система переходит в обычную (скалярную) систему ЯО [10].

В работе [31] $(2+1)$-мерный вариант системы (27) рассматривался в рамках метода Хироты. Была получена система трех билинейных уравнений и найдены ее решения в терминах определителей Вронского. Решения, выражающиеся через определители Грама, были выведены в статье [32].

В завершение этого раздела обсудим вопрос, связанный со временем жизни неравновесной населенности спиновых подуровней парамагнитных ионов, необходимой для достижения условия РЗБ в акустике. Важно, чтобы неравновесное состояние не разрушилось до формирования акустического импульса. Обычно импульсы формируются при распространении на расстояние порядка дисперсионной длины $z_{\mathrm{d}}=\tau_{\mathrm{p}}^{2} / \beta=\Delta \tau_{\mathrm{p}}^{2} / \alpha$ (см. уравнения (27)). Как видно из формулы $(25)$, условие РЗБ $v_{\mathrm{g}}=a_{\|}$выполняется, если $\alpha=1 / a_{\perp}-1 / a_{\|}$. Тогда $z_{\mathrm{d}}=\Delta \tau_{\mathrm{p}}^{2} a_{\|} a_{\perp} /\left(a_{\|}-a_{\perp}\right)$. Этой длине соответствует время распространения

$$
t_{\mathrm{d}}=\frac{z_{\mathrm{d}}}{a_{\|}}=\Delta \tau_{\mathrm{p}}^{2} \frac{a_{\perp}}{a_{\|}-a_{\perp}} .
$$

Взяв $\Delta \tau_{\mathrm{p}} \sim 10$ и $a_{\|} \approx 1.1 a_{\perp}$, найдем $t_{\mathrm{d}} \sim 10^{2} \tau_{\mathrm{p}}$. При $\Delta \sim 10^{9} \mathrm{c}^{-1}$ и, соответственно, $\tau_{\mathrm{p}} \sim 10^{-8}$ с имеем $t_{\mathrm{d}} \sim 10^{-6}$ с, что на несколько порядков меньше времени жизни $T_{1}$ парамагнитных ионов в неравновесном состоянии при температуре жидкого гелия [18], [23]. Поэтому на временах формирования импульса можно считать $T_{1}=\infty$ и не учитывать неустойчивость неравновесного состояния. 


\section{4. ПРЕДСТАВЛЕНИЕ НУЛЕВОЙ КРИВИЗНЫ. ИЕРАРХИИ РЕШЕНИЙ}

Введем новые независимые и зависимые переменные

$$
\begin{gathered}
x=-\frac{\alpha b}{2 \beta^{2}} T, \quad \tau=\frac{\alpha^{2} b^{2}}{4 \beta^{3}} z, \\
\Omega_{1}=\frac{2 \beta^{2}}{\alpha b} \Phi_{+}, \quad \Omega_{2}=\frac{2 \beta^{2}}{\alpha b} \Phi_{-}, \quad u=\frac{4 \beta^{3}}{\alpha b^{2}} U .
\end{gathered}
$$

В терминах этих переменных двухкомпонентная система ЯО (27) примет вид

$$
\begin{aligned}
i \frac{\partial \Omega_{1}}{\partial \tau} & =\frac{\partial^{2} \Omega_{1}}{\partial x^{2}}+u \Omega_{1}, \\
i \frac{\partial \Omega_{2}}{\partial \tau} & =\frac{\partial^{2} \Omega_{2}}{\partial x^{2}}+u \Omega_{2}, \\
\frac{\partial u}{\partial \tau} & =2 \frac{\partial}{\partial x}\left(\left|\Omega_{1}\right|^{2}+\left|\Omega_{2}\right|^{2}\right) .
\end{aligned}
$$

Система уравнений (28) и скалярная система ЯО являются частными случаями $N$-компонентной системы ЯО

$$
\begin{aligned}
i \frac{\partial \Omega_{j}}{\partial \tau} & =\frac{\partial^{2} \Omega_{j}}{\partial x^{2}}+u \Omega_{j}, \quad j=1, \ldots, N, \\
\frac{\partial u}{\partial \tau} & =2 \frac{\partial}{\partial x} \sum_{j=1}^{N}\left|\Omega_{j}\right|^{2}
\end{aligned}
$$

при $N=2$ и $N=1$ соответственно. Систему (29) можно записать как условие нулевой кривизны

$$
\frac{\partial \hat{L}(\lambda)}{\partial \tau}-\frac{\partial \hat{A}(\lambda)}{\partial x}+[\hat{L}(\lambda), \hat{A}(\lambda)]=0
$$

в котором матрицы $\hat{L}(\lambda)$ и $\hat{A}(\lambda)$ размера $(N+2) \times(N+2)$ определены следующим образом:

$$
\begin{aligned}
\hat{L}(\lambda) & =\left(\begin{array}{ccccc}
-\lambda & \Omega_{1} & \ldots & \Omega_{N} & i u \\
0 & 0 & \ldots & 0 & -\Omega_{1}^{*} \\
\vdots & \vdots & \ddots & \vdots & \vdots \\
0 & 0 & \ldots & 0 & -\Omega_{N}^{*} \\
i & 0 & \ldots & 0 & \lambda
\end{array}\right), \\
\hat{A}(\lambda) & =i\left(\begin{array}{cccccc}
-\lambda^{2} & \lambda \Omega_{1}-\frac{\partial \Omega_{1}}{\partial x} & \ldots & \lambda \Omega_{N}-\frac{\partial \Omega_{N}}{\partial x} & \sum_{j=1}^{N}\left|\Omega_{j}\right|^{2} \\
i \Omega_{1}^{*} & 0 & \ldots & 0 & \lambda \Omega_{1}^{*}-\frac{\partial \Omega_{1}^{*}}{\partial x} \\
\vdots & \vdots & \ddots & \vdots & \vdots \\
i \Omega_{N}^{*} & 0 & \ldots & 0 & \lambda \Omega_{N}^{*}-\frac{\partial \Omega_{N}^{*}}{\partial x} \\
0 & -i \Omega_{1} & \ldots & -i \Omega_{N} & -\lambda^{2}
\end{array}\right),
\end{aligned}
$$


где $\lambda$ - спектральный параметр. Таким образом, векторные системы ЯО (28) и (29) интегрируемы в рамках МОЗР.

Равенство (30) является условием совместности переопределенной системы линейных уравнений (пары Лакса)

$$
\frac{\partial \psi}{\partial x}=\hat{L}(\lambda) \psi, \quad \frac{\partial \psi}{\partial \tau}=\hat{A}(\lambda) \psi
$$

где $\psi=\psi(x, \tau, \lambda)=\left(\psi_{1}, \ldots, \psi_{N+2}\right)^{\mathrm{T}}$ - векторное решение со спектральным параметром $\lambda$.

Введем также сопряженную пару Лакса

$$
\frac{\partial \xi}{\partial x}=-\xi \hat{L}(\varkappa), \quad \frac{\partial \xi}{\partial \tau}=-\xi \hat{A}(\varkappa)
$$

где $\xi=\xi(x, \tau, \varkappa)=\left(\xi_{1}, \ldots, \xi_{N+2}\right)$ - векторное решение со спектральным параметром $\varkappa$. Ее условием совместности также является равенство (30).

Матрицы $\hat{L}(\lambda)$ и $\hat{A}(\lambda)$ имеют особый вид, т. е. на них наложены редукционные ограничения, уменьшающие число независимых элементов матриц. Эти редукции задают свойства симметрии (автоморфизмы) в пространствах решений пар Лакса [33]. Рассмотрим эти свойства симметрии подробнее.

Пусть вектор-столбец $\varphi=\left(\varphi_{1}, \ldots, \varphi_{N+2}\right)^{\mathrm{T}}-$ решение пары Лакса $(31)$ при $\lambda=\nu$. Мы считаем, что $u \in \mathbb{R}$, поэтому вектор-строка $\varphi^{\dagger} C$, где

$$
C=\left(\begin{array}{ccccc}
0 & 0 & \ldots & 0 & 1 \\
0 & 1 & \ldots & 0 & 0 \\
\vdots & \vdots & \ddots & \vdots & \vdots \\
0 & 0 & \ldots & 1 & 0 \\
1 & 0 & \ldots & 0 & 0
\end{array}\right)
$$

является решением сопряженной пары Лакса (32) при $\varkappa=\nu^{*}$. Это свойство симметрии следует из редукционных ограничений

$$
\hat{L}^{\dagger}\left(\lambda^{*}\right) C+C \hat{L}(\lambda)=0, \quad \hat{A}^{\dagger}\left(\lambda^{*}\right) C+C \hat{A}(\lambda)=0 .
$$

Существует еще одно свойство симметрии. Если

$$
\varphi=\left(-i \frac{\partial \varphi_{N+2}}{\partial x}+i \nu \varphi_{N+2}, \varphi_{2}, \ldots, \varphi_{N+2}\right)^{\mathrm{T}}
$$

есть векторное решение пары Лакса (31) при $\lambda=\nu$, то векторное решение этой же пары Лакса при $\lambda=-\nu$ можно выбрать в виде

$$
\varphi^{\prime}=\left(-i \frac{\partial \varphi_{N+2}}{\partial x}-i \nu \varphi_{N+2}, \varphi_{2}, \ldots, \varphi_{N+2}\right)^{\mathrm{T}}
$$

Можно показать, что упомянутые выше два свойства симметрии в пространствах решений пар Лакса полностью определяют вид матриц $\hat{L}(\lambda)$ и $\hat{A}(\lambda)$. 
Обсудим вопрос о построении бесконечных иерархий решений (в том числе многосолитонных решений) векторных систем ЯО (28) и (29). Такие иерархии можно получить, используя, например, технику преобразования Дарбу (ПД) [34]. Удобство этой техники заключается в том, что она позволяет учесть редукционные ограничения, наложенные на коэффициенты пар Лакса. Преобразованные коэффициенты также будут удовлетворять редукциям, если проводить последовательность ПД на решениях пар Лакса, связанных свойствами симметрии [35]. Так, редукционные ограничения, задаваемые равенствами (33), будут иметь место для преобразованных коэффициентов, если выполнить так называемое бинарное ПД (БПД), в котором преобразованные решения $\tilde{\psi}$ и $\tilde{\xi}$ прямой и сопряженной пар Лакса определены следующим образом:

$$
\tilde{\psi}=\left(E-\frac{\nu-\nu^{*}}{\lambda-\nu^{*}} \frac{\varphi \varphi^{\dagger} C}{\left(\varphi^{\dagger} C, \varphi\right)}\right) \psi, \quad \tilde{\xi}=\xi\left(E-\frac{\nu-\nu^{*}}{\nu-\varkappa} \frac{\varphi \varphi^{\dagger} C}{\left(\varphi^{\dagger} C, \varphi\right)}\right)
$$

где $E$ - единичная матрица, $\left(\varphi^{\dagger} C, \varphi\right)=\varphi_{1} \varphi_{N+2}^{*}+\varphi_{1}^{*} \varphi_{N+2}+\sum_{j=2}^{N-1}\left|\varphi_{j}\right|^{2}-$ скалярное произведение вектор-строки $\varphi^{\dagger} C$ на вектор-столбец $\varphi$. Здесь выражение $\varphi^{\dagger} C$ соответствует такому выбору решения сопряженной пары Лакса (32), при котором сохраняется редукция (33) после проведения БПД.

Проведение двух подобных БПД решения $\varphi$ вида (34) и решения $\varphi^{\prime}$ вида (35) позволяет сохранить оба свойства симметрии в пространствах решений пар Лакса, которые соответствуют векторной системе ЯО. При этом получаются следующие формулы преобразований:

$$
\begin{array}{rlrl}
\psi_{1}[1] & =i \lambda \psi_{N+2}[1]-i \frac{\partial \psi_{N+2}[1]}{\partial x}, & \\
\psi_{j}[1] & =\psi_{j}-\frac{\delta\left(\varphi^{\dagger} C, \psi\right)}{\delta\left(\varphi^{\dagger} C, \varphi\right)} \varphi_{j}, & & \\
\xi_{j}[1] & =\xi_{j}-\frac{\delta(\xi, \varphi)}{\delta\left(\varphi^{\dagger} C, \varphi\right)}\left(\varphi^{\dagger} C\right)_{j}, & & j=1, \ldots, N+2, N+1, \\
\xi_{N+2}[1] & =-i \varkappa \xi_{1}[1]+i \frac{\partial \xi_{1}[1]}{\partial x}, &
\end{array}
$$

И

$$
\begin{aligned}
\Omega_{j}[1] & =\Omega_{j}+\frac{\varphi_{j+1}^{*} \varphi_{N+2}}{\delta\left(\varphi^{\dagger} C, \varphi\right)}, \quad j=1, \ldots, N, \\
u[1] & =u+2 \frac{\partial^{2}}{\partial x^{2}} \ln \delta\left(\varphi^{\dagger} C, \varphi\right),
\end{aligned}
$$

Для удобства записи мы использовали здесь обозначения

$$
\delta(\xi, \psi)=\frac{1}{\lambda^{2}-\varkappa^{2}}\left(\xi_{1} \frac{\partial \psi_{N+2}}{\partial x}-\psi_{N+2} \frac{\partial \xi_{1}}{\partial x}+i \sum_{j=2}^{N+1} \xi_{j} \psi_{j}\right)
$$


а также

$$
\delta\left(\varphi^{\dagger} C, \varphi\right)=\frac{1}{\nu^{2}-\nu^{* 2}}\left(\varphi_{N+2}^{*} \frac{\partial \varphi_{N+2}}{\partial x}-\varphi_{N+2} \frac{\partial \varphi_{N+2}^{*}}{\partial x}+i \sum_{j=2}^{N+1}\left|\varphi_{j}\right|^{2}\right) .
$$

Соотношения (37) определяют новое (преобразованное) решение векторной системы ЯО (29). При этом равенства (36) определяют соответствующие решения пар Лакса, другими словами, вектор-столбец $\psi[1]=\left(\psi_{1}[1], \ldots, \psi_{N+2}[1]\right)^{\mathrm{T}}$ и вектор-строка $\xi[1]=\left(\xi_{1}[1], \ldots, \xi_{N+2}[1]\right)$ являются решениями переопределенных систем, которые получаются из систем (31) и (32) при помощи замен

$$
\psi \rightarrow \psi[1], \quad \xi \rightarrow \xi[1], \quad \hat{L}(\lambda) \rightarrow \hat{L}(\lambda)[1], \quad \hat{A}(\lambda) \rightarrow \hat{A}(\lambda)[1]
$$

где подстановки $u \rightarrow u[1], \Omega_{j} \rightarrow \Omega_{j}[1], j=1,2, \ldots, N$, переводят матрицы $\hat{L}(\lambda), \hat{A}(\lambda)$ в матрицы $\hat{L}(\lambda)[1], \hat{A}(\lambda)[1]$.

Пусть $\varphi^{(m)}=\left(\varphi_{1}^{(m)}, \ldots, \varphi_{N+2}^{(m)}\right)^{\mathrm{T}}-$ решения пары Лакса $(31)$ при $\lambda=\nu_{m}, m=$ $1, \ldots, M$. Так как имеет место тождество

$$
\delta(\xi[1], \psi[1]) \delta\left(\varphi^{\dagger} C, \varphi\right)=\left|\begin{array}{ll}
\delta(\xi, \psi) & \delta\left(\varphi^{\dagger} C, \psi\right) \\
\delta(\xi, \varphi) & \delta\left(\varphi^{\dagger} C, \varphi\right)
\end{array}\right|
$$

$M$-кратная итерация ПД (36), (37) дает следующие выражения для преобразованных решений векторной системы ЯО (29):

$$
\begin{aligned}
\Omega_{j}[M] & =\Omega_{j}+\frac{1}{D[M]} \sum_{m, k=1}^{M} D^{(m, k)}[M] \varphi_{j+1}^{(m)^{*}} \varphi_{N+2}^{(k)}, \quad j=1, \ldots, N, \\
u[M] & =u+2 \frac{\partial^{2}}{\partial x^{2}} \ln D[M]
\end{aligned}
$$

где

$$
D[M]=\left|\begin{array}{ccc}
\delta\left(\varphi^{(1)^{\dagger}} C, \varphi^{(1)}\right) & \ldots & \delta\left(\varphi^{(1)^{\dagger}} C, \varphi^{(M)}\right) \\
\vdots & \ddots & \vdots \\
\delta\left(\varphi^{(M)^{\dagger}} C, \varphi^{(1)}\right) & \ldots & \delta\left(\varphi^{(M)^{\dagger}} C, \varphi^{(M)}\right)
\end{array}\right|
$$

а $D^{(m, k)}[M]$ - алгебраическое дополнение элемента матрицы определителя $D[M]$, расположенного в $m$-й строке и $k$-м столбце.

Если за исходное решение векторной системы ЯО (29) взят нулевой фон,

$$
\Omega_{1}=\cdots=\Omega_{N}=u=0,
$$

то формулы (37) и (38) позволяют получить соответственно ее одно- и $M$-солитонные решения. 

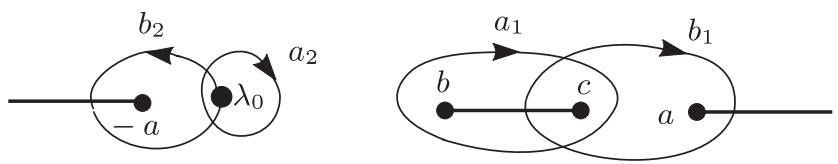

Рис. 2. Поверхность Глп в случае частичного вырождения. Вещественные точки ветвления.

В качестве иллюстрации упомянутой в разделе 1 устойчивости солитонов построим решение системы (29) на нетривиальном фоне. Пусть $\Omega_{1}=\cdots=\Omega_{N}=0$, $u=u(x)$. Тогда из пары Лакса (31) следует, что $\psi_{j}=a_{j}$ - некоторые постоянные при $j=2, \ldots, N+1$, а $\psi_{N+2}-$ решение линейного уравнения Шредингера

$$
\frac{\partial^{2} \psi_{N+2}}{\partial x^{2}}+u \psi_{N+2}=\lambda^{2} \psi_{N+2}
$$

и уравнения

$$
\frac{\partial \psi_{N+2}}{\partial \tau}=-i \lambda^{2} \psi_{N+2}
$$

Положим $u=u_{g}(x)$, где $u_{g}(x)-g$-зонный потенциал уравнения (39). Так как он является стационарным решением соответствующего высшего уравнения иерархии КдФ, решение системы уравнений (39), (40) можно выразить в терминах этого потенциала [36]:

$$
\psi_{N+2}=\sqrt{F_{g}}\left(a_{+} \exp \left[-i \lambda^{2} \tau+\varepsilon_{g} \int \frac{d x}{F_{g}}\right]+a_{-} \exp \left[-i \lambda^{2} \tau-\varepsilon_{g} \int \frac{d x}{F_{g}}\right]\right) .
$$

Здесь $a_{+}$и $a_{-}-$произвольные постоянные, $b_{0}=f_{0}=1, f_{1}=u_{g}(x) / 2$,

$$
\begin{gathered}
F_{g}=\sum_{n=0}^{g} \sum_{j=0}^{g-n} \lambda^{2 n} b_{j} f_{g-n-j}, \\
f_{k}=\frac{1}{8} \sum_{j=1}^{k-1}\left(2 \frac{d^{2} f_{j}}{d x^{2}} f_{k-j-1}-\frac{d f_{j}}{d x} \frac{d f_{k-j-1}}{d x}-4 f_{j} f_{k-j}+4 u_{g}(x) f_{j} f_{k-j-1}\right)+\frac{1}{2} u_{g}(x) f_{k-1}
\end{gathered}
$$

для $k=2, \ldots, g$, постоянная $\varepsilon_{g}$ определяется равенством

$$
\varepsilon_{g}^{2}=-\frac{1}{2} F_{g} \frac{d^{2} F_{g}}{d x^{2}}+\frac{1}{4}\left(\frac{d F_{g}}{d x}\right)^{2}+\left(\lambda^{2}-u_{g}(x)\right) F_{g}^{2},
$$

постоянные $b_{j}, j=1, \ldots, g$, задают спектральную кривую.

Возьмем в качестве исходного потенциала линейного уравнения Шредингера (39) однозонный потенциал (кноидальную волну)

$$
u_{1}(x)=\left(\gamma_{1}-\gamma_{2}\right) \operatorname{cn}^{2}\left(\sqrt{\frac{\gamma_{1}-\gamma_{3}}{2}} x ; \sqrt{\frac{\gamma_{1}-\gamma_{2}}{\gamma_{1}-\gamma_{3}}}\right)+\gamma_{2},
$$



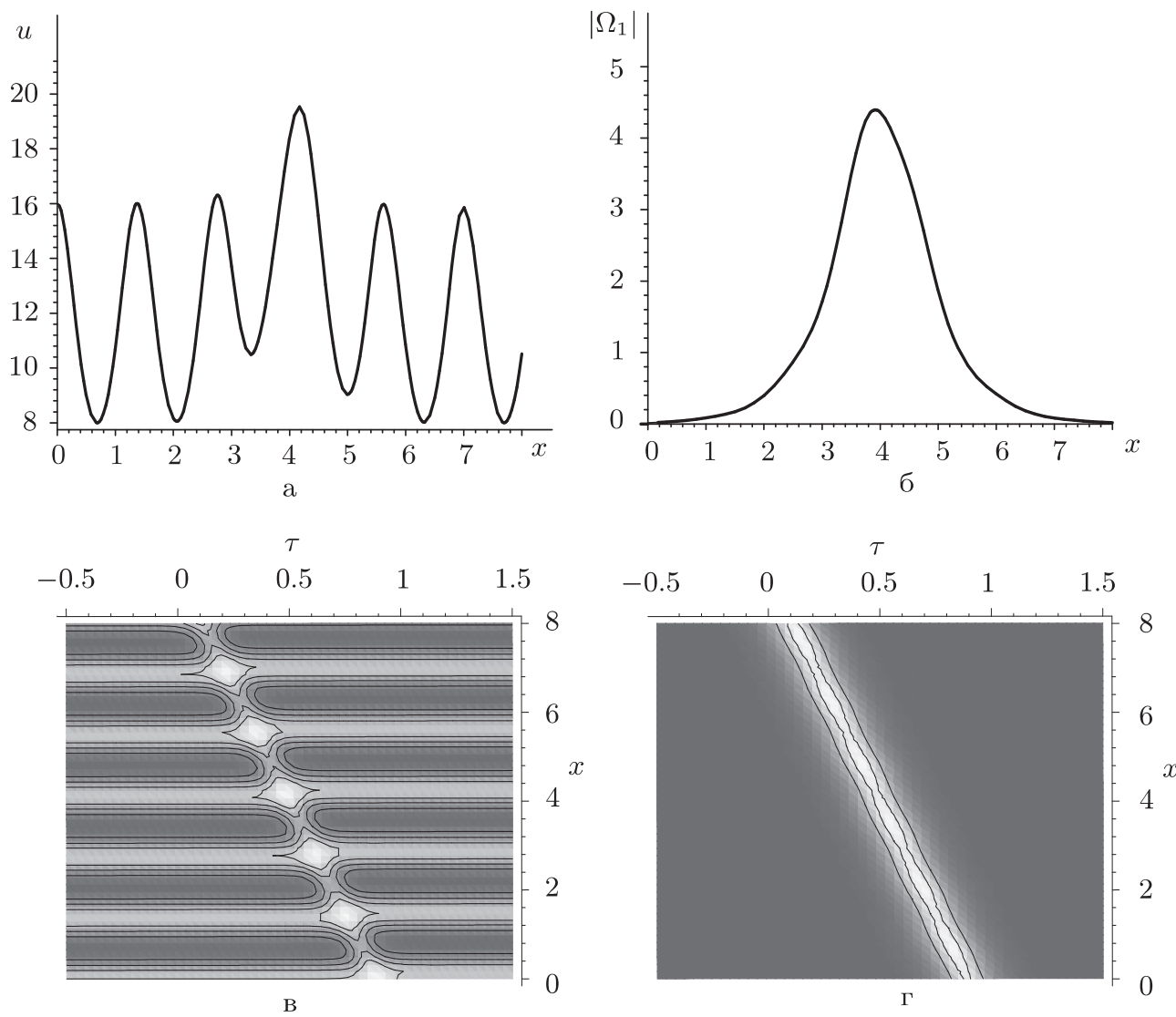
0.5
0.7
0.9

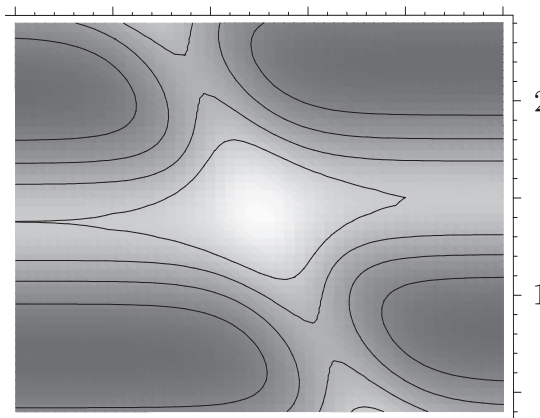

д

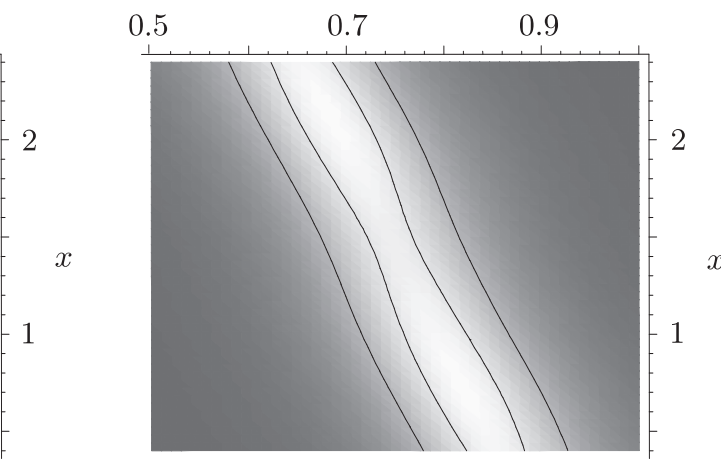

e

Рис. 3. Решения $u\left(\mathrm{a}\right.$, в, д) и $\left|\Omega_{1}\right|(б, г$, е) для значений параметров $\nu=2+4 i$, $\gamma_{1}=16, \gamma_{2}=8, \gamma_{3}=1, a_{2}=2, a_{3}=1, a_{4}=\cdots=a_{N}=0$. Графики на рис. "а" и "б" построены при $\tau=0.5$. Более светлый оттенок серого цвета на двумерных графиках "в" и “г”, а также на их увеличенных фрагментах "д" и "е" соответствует бо́льшим значениям переменных $u$ и $\left|\Omega_{1}\right|$. 
где $\gamma_{1}, \gamma_{2}, \gamma_{3}$ - вещественные постоянные, $\gamma_{1}>\gamma_{2}>\gamma_{3}$. Выражение для компоненты $\varphi_{N+2}$ решения пары Лакса при $\lambda=\nu$ следует из формулы (41). Тогда ПД (37) дает следующее несингулярное решение векторной системы ЯО (29):

$$
\Omega_{j}=\frac{a_{j+1}^{*} \theta}{\delta}, \quad j=1, \ldots, N, \quad u=u_{1}(x)+2 \frac{\partial^{2}}{\partial x^{2}} \ln \delta,
$$

где

$$
\begin{gathered}
\delta=\frac{1}{\nu^{2}-\nu^{* 2}}\left(\theta^{*} \frac{\partial \theta}{\partial x}-\theta \frac{\partial \theta^{*}}{\partial x}+i \sum_{j=2}^{N+1}\left|a_{j}\right|^{2}\right) \\
\theta=\sqrt{F_{1}} \exp \left[-i \nu^{2} \tau+\varepsilon_{1} \int \frac{d x}{F_{1}}\right], \quad F_{1}=\nu^{2}+\frac{u_{1}(x)-\gamma_{1}-\gamma_{2}-\gamma_{3}}{2} \\
\varepsilon_{1}=\sqrt{\left(\nu^{2}-\frac{\gamma_{1}+\gamma_{2}}{2}\right)\left(\nu^{2}-\frac{\gamma_{2}+\gamma_{3}}{2}\right)\left(\nu^{2}-\frac{\gamma_{3}+\gamma_{1}}{2}\right)}
\end{gathered}
$$

Решение (42) описывает распространение солитона векторной системы ЯО на фоне кноидальной волны. Соответствующие графики длинноволновой компоненты $u$ и абсолютной величины коротковолновой компоненты $\Omega_{1}$ представлены на рис. 3. Смещение линий уровня при $\tau \rightarrow \infty$ относительно линий при $\tau \rightarrow-\infty$ на рис. Зв и Зд означает фазовый сдвиг горбов кноидальной волны в результате взаимодействия с солитоном. Периодическое изменение наклона линий уровня на рис. Зг и Зе указывает на изменение групповой скорости солитона и на его фазовый сдвиг при взаимодействии с каждым горбом кноидальной волны. Также это указывает на обмен энергией между коротковолновыми и длинноволновой компонентами солитона.

\section{5. СТАЦИОНАРНАЯ И АВТОМОДЕЛЬНАЯ РЕДУКЦИИ}

Этот раздел посвящен системам обыкновенных дифференциальных уравнений, возникающим как редукции векторной системы ЯО (29).

Рассмотрим стационарную редукцию системы (29). Пусть $x_{\mathrm{s}}=x-v \tau$, где $v-$ вещественная постоянная, и $u=u\left(x_{\mathrm{s}}\right)$. Тогда можно положить

$$
\Omega_{j}=\sqrt{\frac{|v|}{2}} q_{j} \exp \left[-\frac{i v}{2} x+i\left(\frac{v^{2}}{4}-\gamma-c_{j}\right) \tau\right],
$$

где $q_{j}=q_{j}\left(x_{\mathrm{s}}\right), \gamma$ и $c_{j}$ - произвольные вещественные постоянные, $j=1, \ldots, N$. Из последнего уравнения системы (29) после интегрирования находим

$$
u=-\sigma \sum_{j=1}^{N}\left|q_{j}\right|^{2}+\gamma .
$$

Здесь $\sigma=\operatorname{sgn} v$; постоянная интегрирования взята равной $\gamma$ без потери общности. Оставшиеся уравнения системы (29) дают систему обыкновенных дифференциальных уравнений

$$
\frac{d^{2} q_{j}}{d x_{\mathrm{s}}^{2}}=\left(c_{j}+\sigma \sum_{j=1}^{N}\left|q_{j}\right|^{2}\right) q_{j}, \quad j=1, \ldots, N
$$


Полученные уравнения представляют собой комплексный вариант системы Гарнье [37], [38] и содержат два неэквивалентных случая, соответствующих разным знакам $\sigma$.

Систему (43) можно записать в виде условия совместности

$$
\frac{\partial \hat{A}_{\mathrm{s}}(\lambda)}{\partial x_{\mathrm{s}}}+\left[\hat{A}_{\mathrm{s}}(\lambda), \hat{L}_{\mathrm{s}}(\lambda)\right]=0
$$

в котором

$$
\begin{aligned}
& \hat{L}_{\mathrm{s}}(\lambda)=\left(\begin{array}{ccccc}
-\lambda_{-} & q_{1} & \ldots & q_{N} & i \gamma-i \sigma \sum_{j=1}^{N}\left|q_{j}\right|^{2} \\
0 & 0 & \ldots & 0 & -q_{1}^{*} \\
\vdots & \vdots & \ddots & \vdots & \vdots \\
0 & 0 & \ldots & 0 & -q_{N}^{*} \\
i & 0 & \ldots & 0 & \lambda_{+}
\end{array}\right) \\
& \hat{A}_{\mathrm{S}}(\lambda)=i\left(\begin{array}{ccccc}
\lambda_{-}^{2}-\gamma & \frac{d q_{1}}{d x_{\mathrm{s}}}-\lambda_{-} q_{1} & \ldots & \frac{d q_{N}}{d x_{\mathrm{s}}}-\lambda_{-} q_{N} & \sum_{j=1}^{N}\left|q_{j}\right|^{2}-2 \sigma \gamma \\
-i q_{1}^{*} & c_{1} & \ldots & 0 & \frac{d q_{1}^{*}}{d x_{\mathrm{s}}}-\lambda_{+} q_{1}^{*} \\
\vdots & \vdots & \ddots & \vdots & \vdots \\
-i q_{N}^{*} & 0 & \ldots & c_{N} & \frac{d q_{N}^{*}}{d x_{\mathrm{s}}}-\lambda_{+} q_{N}^{*} \\
-2 \sigma & i q_{1} & \ldots & i q_{N} & \lambda_{+}^{2}-\gamma
\end{array}\right),
\end{aligned}
$$

где $\lambda_{ \pm}=\lambda \pm i \sigma$. Заметим, что здесь размерность матриц на единицу больше по сравнению с обычным представлением для системы Гарнье, которое следует из представления Лакса для НУШ [38]. Кроме того, матрицы $\hat{L}_{\mathrm{s}}(\lambda)$ и $\hat{A}_{\mathrm{s}}(\lambda)$ содержат произвольный параметр $\gamma$, не входящий в систему (43). От этого параметра можно избавиться, исключив одну из компонент векторного решения соответствующей переопределенной системы и заменив спектральный параметр.

Система (43) имеет первые интегралы

$$
\begin{aligned}
F_{j} & =\frac{d q_{j}}{d x_{\mathrm{s}}} \frac{d q_{j}^{*}}{d x_{\mathrm{s}}}-c_{j}\left|q_{j}\right|^{2}-\frac{\sigma}{2}\left|q_{j}\right|^{2} \sum_{k=1}^{N}\left|q_{k}\right|^{2}+\frac{\sigma}{2} \sum_{\substack{1 \leqslant k \leqslant N \\
k \neq j}} \frac{1}{c_{k}-c_{j}}\left|q_{j} \frac{d q_{k}}{d x_{\mathrm{s}}}-q_{k} \frac{d q_{j}}{d x_{\mathrm{s}}}\right|^{2}, \\
i_{j} & =q_{j} \frac{d q_{j}^{*}}{d x_{\mathrm{s}}}-q_{j}^{*} \frac{d q_{j}}{d x_{\mathrm{s}}}, \quad j=1, \ldots, N
\end{aligned}
$$

Также ее первыми интегралами являются собственные значения матрицы $\hat{A}_{\mathrm{s}}(\lambda)$ или, очевидно, коэффициенты характеристического многочлена $\operatorname{det}\left(\hat{A}_{\mathrm{s}}(\lambda)-\Lambda E\right)$ при различных степенях $\Lambda$ и $\lambda$. Один из таких коэффициентов дает интеграл

$$
I=\frac{1}{2} \sum_{j=1}^{N}\left(\frac{d q_{j}}{d x_{\mathrm{s}}} \frac{d q_{j}^{*}}{d x_{\mathrm{s}}}-c_{j}\left|q_{j}\right|^{2}\right)-\frac{1}{4 \sigma}\left(\sum_{j=1}^{N}\left|q_{j}\right|^{2}\right)^{2} .
$$


При наложении редукционных ограничений $q_{j} \in \mathbb{R}, j=1, \ldots, N$, интеграл (44) переходит в гамильтониан соответствующей системы обыкновенных дифференциальных уравнений. При этом производные $q_{j}$ по $x_{\mathrm{s}}$ дают канонически сопряженные переменные. Возникающую в этом случае систему можно рассматривать как систему уравнений, описывающую ньютоновскую динамику частицы в $N$-мерном простран-

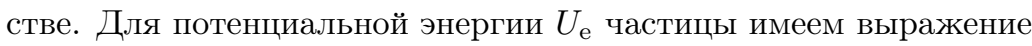

$$
U_{\mathrm{e}}=-\sum_{j=1}^{N} c_{j} q_{j}^{2}-\frac{1}{4 \sigma}\left(\sum_{j=1}^{N} q_{j}^{2}\right)^{2} .
$$

Видно, что финитные движения невозможны, только если постоянные $c_{j}>0$ при всех $j=1, \ldots, N$ и $\sigma>0$.

Рассмотрим теперь автомодельную редукцию векторной системы ЯО (29). Положим $s=\operatorname{sgn} \tau$. Введем новые переменные $\zeta, v_{j}=v_{j}(\zeta), j=1, \ldots, N$, и $w=w(\zeta)$ следующим образом:

$$
\zeta=\frac{(s \tau)^{-1 / 2} x}{\sqrt{2}}, \quad \Omega_{j}=\frac{(s \tau)^{-3 / 4+i C_{j} / 2}}{2^{3 / 4}} v_{j}, \quad u=-\frac{w}{2 \tau},
$$

где $C_{j}$ - вещественные постоянные. В (45) мы считаем корни $(s \tau)^{-1 / 2}$ и $(s \tau)^{-1 / 4}$ арифметическими, а $\left|(s \tau)^{i C_{j} / 2}\right|=1$. Тогда из (29) получим систему обыкновенных дифференциальных уравнений

$$
\begin{aligned}
\frac{d^{2} v_{j}}{d \zeta^{2}} & =i \zeta \frac{d v_{j}}{d \zeta}+\left(C_{j}+s w+\frac{3}{2} i\right) v_{j}, \quad j=1, \ldots, N \\
\frac{d w}{d \zeta} & =\frac{2}{\zeta}\left(\frac{d}{d \zeta} \sum_{j=1}^{N}\left|v_{j}\right|^{2}-w\right) .
\end{aligned}
$$

Кроме того, эту систему можно получить из векторной системы ЯО при более общей замене переменных, которая следует из формул (45) при учете сдвигов по координатам $\tau \rightarrow \tau+\tau_{0}, x \rightarrow x+x_{0}$, где $\tau_{0}$ и $x_{0}$ - вещественные постоянные. Отметим, что системы уравнений, получающиеся из (46) при разных знаках параметра $s$, неэквивалентны.

Система уравнений (46) тоже является условием совместности

$$
\frac{\partial \widehat{P}(\lambda)}{\partial \lambda}-\frac{\partial \widehat{Q}(\lambda)}{\partial \zeta}+[\widehat{P}(\lambda), \widehat{Q}(\lambda)]=0
$$

переопределенной линейной системы $(P-Q$ пары) с матрицами $\widehat{P}(\lambda)$ и $\widehat{Q}(\lambda)$ вида

$$
\widehat{P}(\lambda)=\left(\begin{array}{ccccc}
-\lambda & v_{1} & \ldots & v_{N} & i w \\
0 & 0 & \ldots & 0 & -v_{1}^{*} \\
\vdots & \vdots & \ddots & \vdots & \vdots \\
0 & 0 & \ldots & 0 & -v_{N}^{*} \\
-s i & 0 & \ldots & 0 & \lambda
\end{array}\right), \quad \widehat{Q}(\lambda)=\lambda \widehat{Q}_{1}+\widehat{Q}_{0}+\frac{\widehat{Q}_{-1}}{\lambda},
$$

где

$$
\widehat{Q}_{1}=\operatorname{diag}(i, 0, \ldots, 0, i)
$$




$$
\begin{aligned}
\widehat{Q}_{0}= & \left(\begin{array}{ccccc}
-\zeta & -i v_{1} & \ldots & -i v_{N} & 0 \\
0 & 0 & \ldots & 0 & -i v_{1}^{*} \\
\vdots & \vdots & \ddots & \vdots & \vdots \\
0 & 0 & \ldots & 0 & -i v_{N}^{*} \\
0 & 0 & \ldots & 0 & \zeta
\end{array}\right), \\
\widehat{Q}_{-1}= & \left(\begin{array}{ccccc}
\frac{1}{2} & i \frac{d v_{1}}{d \zeta}+\zeta v_{1} & \ldots & i \frac{d v_{N}}{d \zeta}+\zeta v_{N} & i \zeta w-i \sum_{j=1}^{N}\left|v_{j}\right|^{2} \\
-s v_{1}^{*} & i C_{1} & \ldots & 0 & i \frac{d v_{1}^{*}}{d \zeta}-\zeta v_{1}^{*} \\
\vdots & \vdots & \ddots & \vdots & \vdots \\
-s v_{N}^{*} & 0 & \ldots & i C_{N} & i \frac{d v_{N}^{*}}{d \zeta}-\zeta v_{N}^{*} \\
-s i \zeta & s i v_{1} & \ldots & s i v_{N} & -\frac{1}{2}
\end{array}\right) .
\end{aligned}
$$

Коэффициент при $\lambda^{-1}$ в условии совместности (47) задает уравнение для матрицы $\widehat{Q}_{-1}$ :

$$
\frac{d \widehat{Q}_{-1}}{d \zeta}=\left[\widehat{P}(0), \widehat{Q}_{-1}\right]
$$

Отсюда следует, что собственные значения этой матрицы не зависят от переменной $\zeta$. Запишем характеристический многочлен матрицы $Q_{-1}$ следующим образом:

$$
\operatorname{det}\left(\widehat{Q}_{-1}-\Lambda E\right)=(-1)^{N}\left(\Lambda^{N+2}-i \sum_{j=1}^{N} C_{j} \Lambda^{N+1}-\sum_{m=0}^{N} i^{m} I_{m+1} \Lambda^{N-m}\right) .
$$

Вещественные коэффициенты $I_{m}$ характеристического многочлена являются, очевидно, первыми интегралами системы (46). Первый из этих интегралов

$$
I_{1}=-s \zeta^{2} w+3 s \zeta \sum_{j=1}^{N}\left|v_{j}\right|^{2}-i s \sum_{j=1}^{N}\left(v_{j} \frac{d v_{j}^{*}}{d \zeta}-v_{j}^{*} \frac{d v_{j}}{d \zeta}\right)-\frac{1}{2} \sum_{\substack{1 \leqslant j, m \leqslant N, m \neq j}} C_{j} C_{m}-\frac{1}{4}
$$

позволяет исключить переменную $w$ из уравнений (46). Заметим, что выражение для $w$, получающееся при этом, не зависит по сути от знака параметра $s$.

\section{6. ЗАКЛЮЧЕНИЕ}

В настоящей работе исследовано распространение векторного продольно-поперечного упругого импульса в кристалле, содержащем парамагнитные примеси. Внешняя статическая деформация кристалла и внешнее магнитное поле создают при наложенных условиях и в геометрии Фарадея схему спин-фононных переходов, изображенную на рис. 1.

Подчеркнем, что похожая задача возникает в квазирезонансной оптике [22]. Но там роль длинноволновой компоненты играет терагерцовый электромагнитный импульс, из-за чего достичь выполнения условия РЗБ (1) относительно просто. Дело 
в том, что фазовая скорость в терагерцовом диапазоне частот обычно меньше групповой скорости в оптическом диапазоне, следовательно, квазирезонансные квантовые частицы, приготовленные изначально в равновесном состоянии, позволяют уменьшить групповую скорость оптических сигналов до значений, удовлетворяющих условию РЗБ.

При равновесной начальной населенности спиновых подуровней в рассмотренной здесь акустической задаче групповая скорость поперечных волн всегда оказывается меньше фазовой скорости продольной волны, т. е. условие РЗБ выполнить невозможно. В итоге взаимодействие между продольным и поперечным звуком слабое, и образование связанных состояний длинно-коротковолнового типа практически неосуществимо. В этом случае импульс остается сугубо поперечным, а его динамика при взаимодействии с парамагнитными примесями в условиях квазирезонанса описывается системой Манакова. Однако если парамагнитные примеси до акустического воздействия приготовлены в неравновесном состоянии, то возникает возможность достичь выполнения условия РЗБ, причем для двух частот одновременно. Тогда при распространении двух поперечных компонент, несущие частоты которых отличаются друг от друга на удвоенную частоту $\omega_{\mathrm{Z}}$ зеемановского расщепления спиновых подуровней, происходит эффективная генерация продольной составляющей и образование связанного длинно-коротковолнового состояния.

Важное обстоятельство состоит в том, что динамика трехкомпонентного продольно-поперечного упругого импульса описывается системой уравнений (27), которая, являясь векторным обобщением системы ЯО, также принадлежит к классу уравнений, интегрируемых в рамках МОЗР. Поэтому ее решения типа уединенных волн являются солитонами в строгом смысле и задаются дискретной частью данных рассеяния. Кроме того, системы обыкновенных дифференциальных уравнений, возникающие как стационарная и автомодельная редукции векторной многокомпонентной системы ЯО (29), тоже представимы в виде условия совместности переопределенных линейных систем, что позволяет сразу найти их первые интегралы. Возможно, что система (46) является новой и заслуживает отдельного рассмотрения с точки зрения свойства Пенлеве [39], наличия преобразований Шлезингера и интегрирования в рамках метода изомонодромных деформаций [40].

Структурная устойчивость решений векторной системы ЯО проиллюстрирована солитонным решением на фоне кноидальной волны. Как оказалось, фазовый сдвиг претерпевают не только солитонный вклад в это решение, но и горбы кноидальной волны.

Коэффициент нелинейности $\alpha$ в рамках принятых нами приближений оказался одним и тем же в уравнениях для коротковолновых компонент $\Phi_{+}$и $\Phi_{-}$. Он пропорционален произведению $G_{\perp}^{2} \omega$, которое представляет собой акустический аналог силы осциллятора оптических переходов. Таким образом, рассмотренный нами квазирезонансный солитонный режим предполагает равенство сил осцилляторов обоих задействованных переходов $1 \leftrightarrow 2$ и $1 \leftrightarrow 3$. Известно, что именно при выполнении этого условия уравнения самоиндуцированной прозрачности, описывающие резонансное распространение двухчастотных оптических импульсов (симултонов) в трехуровневой среде, в которой отсутствует динамический сдвиг частот квантовых 
переходов, интегрируемы в рамках МОЗР [41]. Поэтому представляет интерес исследование на интегрируемость уравнений самоиндуцированной прозрачности, описывающих распространение резонансных (акустических или оптических) импульсов в трехуровневой среде с равными силами осцилляторов переходов в случае, когда имеет место динамический сдвиг частот переходов полем проходящего импульса.

Благодарности. Работа выполнена при финансовой поддержке РФФИ (грант № 13-02-00199_а).

\section{Список литературы}

[1] Л. Д. Фаддеев, УФН, 183:5 (2013), 487-495.

[2] N. J. Zabusky, M. D. Kruskal, Phys. Rev. Lett., 15:6 (1965), 240-243.

[3] В. Е. Захаров, С. В. Манаков, С. П. Новиков, Л. П. Питаевский, Теория солитонов. Метод обратной задачи, Наука, М., 1980; Дж. Л. Лэм, Введение в теорию солитонов, Мир, М., 1983; Л. А. Тахтаджян, Л. Д. Фаддеев, Гамильтонов подход в теории солитонов, Наука, М., 1986.

[4] Р. Додд, Дж. Эйлбек, Дж. Гиббон, Х. Моррис, Солитоны и нелинейные волновые уравнения, Мир, М., 1988.

[5] C. Sulem, P. L. Sulem, The Nonlinear Schrödinger Equation. Self-Focusing and Wave Collapse, Springer, New York, 1999; Н. Н. Ахмедиев, А. Анкевич, Солитонъ. Нелинейнъе импульсы и пучки, Физматлит, М., 2003.

[6] В. Е. Захаров, А. Б. Шабат, ЖКЭТФ, 61:1 (1971), 118-134.

[7] С. В. Манаков, ЖЖЭТФ, 67:2 (1974), 543-555.

[8] П. Г. Крюков, Фемтосекундные импулъсы, Физматлит, М., 2008.

[9] В. Е. Захаров, ЖЭТФ, 62:5 (1972), 1745-1759.

[10] N. Yajima, M. Oikawa, Progr. Theor. Phys., 56:6 (1976), 1719-1739; Y. C. Ma, Stud. Appl. Math., 59:3 (1978), 201-221.

[11] D. J. Benney, Stud. Appl. Math., 56:1 (1977), 81-94.

[12] В. С. Львов, Нелинейные спиновые волны, Наука, М., 1987.

[13] С. В. Сазонов, А. Ф. Соболевский, ЖЭТФ, 123:6 (2003), 1160-1178.

[14] A. N. Bugay, S. V. Sazonov, Phys. Rev. E, 74:6 (2006), 066608, 8 pp.

[15] С. В. Сазонов, Писъма в ЖЭТФ, 81:5 (2005), 259-262.

[16] С. В. Сазонов, Писъма в ЖЭТФ, 96:4 (2012), 281-294.

[17] G. Kh. Kitaeva, Laser Phys. Lett., 5:8 (2008), 559-576.

[18] В. А. Голенищев-Кутузов, В. В. Самарцев, Н. К. Соловаров, Б. М. Хабибулин, Магнитная квантовая акустика, Наука, М., 1977.

[19] Ф. В. Бункин, Ю. А. Кравцов, Г. А. Ляхов, УФН, 149:3 (1986), 391-411.

[20] С. В. Сазонов, Н. В. Устинов, ТМФ, 151:2 (2007), 228-247; 164:2 (2010), 222-242.

[21] А. Н. Бугай, С. В. Сазонов, ЖЭТФ, 139:3 (2011), 464-478.

[22] С. В. Сазонов, Н. В. Устинов, ЖКЭТФ, 142:5(11) (2012), 842-861.

[23] Дж. Такер, Р. Рэмптон, Гиперзвук в физике твердого тела, Мир, М., 1975.

[24] N. S. Shiren, Phys. Rev. B, 2:7 (1970), 2471-2487.

[25] Л. Д. Ландау, Е. М. Лифшиц, Теоретическая физика, т. 7: Теория упругости, Наука, М., 1987.

[26] С. В. Воронков, С. В. Сазонов, ЖКЭТФ, 120:2(8) (2001), 269-279.

[27] С. В. Сазонов, ЖЭТФ, 118:1 (2000), 20-35.

[28] Л. Аллен, Дж. Эберли, Оптический резонанс и двухуровневые атомы, Мир, М., 1978.

[29] M. D. Crisp, Phys. Rev. A, 8:4 (1973), 2128-2135; А. М. Башаров, А. И. Маймистов, Оптика и спектроскопия, 88:3 (2000), 428-434. 
[30] Ч. Киттель, Введение в физику твердого тела, Физматлит, М., 1963.

[31] Y. Ohta, K. Maruno, M. Oikawa, J. Phys. A, 40:27 (2007), 7659-7672, arXiv: nlin/0702051.

[32] T. Kanna, M. Vijayajayanthi, K. Sakkaravarthi, M. Lakshmanan, J. Phys. A, 42:11 (2009), 115103, $20 \mathrm{pp}$.

[33] A. V. Mikhailov, Physica D, 3:1-2 (1981), 73-117.

[34] V.B. Matveev, M. A. Salle, Darboux transformations and solitons, Springer Series in Nonlinear Dynamics, Springer, Berlin, 1991; В. Г. Багров, Б. Ф. Самсонов, ЭЧАЯ, 28 (1997), 951-1012.

[35] N. V. Ustinov, J. Math. Phys., 39:2 (1998), 976-985; J.L. Cieśliński, M. Czachor, N. V. Ustinov, J. Math. Phys., 44:4 (2003), 1763-1780.

[36] Н. В. Устинов, Ю. В. Брежнев, УМН, 57:1(343) (2002), 167-168.

[37] R. Garnier, Rend. Circ. Matem. Palermo, 43:1 (1919), 155-191.

[38] А.М. Переломов, Интегрируемые системы классической механики и алгебры Ли, Наука, М., 1990.

[39] R. Conte (ed.), The Painlevé Property: One Century Later, Springer, New York, 1999.

[40] А. Р. Итс, А. А. Капаев, В. Ю. Новокшенов, А. С. Фокас, Трансценденты Пенлеве. Метод задачи Римана, ИКИ, М., Ижевск, 2005; K. Iwasaki, H. Kimura, S. Shimomura, M. Yoshida, From Gauß to Painlevé. A modern theory of special functions, Aspects of Mathematics, 16, Vieweg \& Sohn., Braunschweig, 1991.

[41] Л. А. Большов, В.В.Лиханский, М.И.Персианцев, ЖЭТФ, 84:3 (1983), 903-911; А. М. Башаров, А. И. Маймистов, ЖЖЭТФ, 94:6(12) (1988), 61-75; H. Steudel, J. Modern Opt., 35:4 (1988), 693-702.

Поступила в редакцию 4.06.2013 